\title{
Module und Formate \\ in Mentoring-Programmen
}

Die klassischen Mentoring-Programme im Bereich der Chancengleicheit sind modular konzipiert und können auf der Basis ihrer drei Bausteine Mentoring-Training-Networking die Mentees auf verschiedenen Ebenen bei der Berufsorientierung und Karriereentwicklung unterstützen.

Während das Modul Mentoring die Beratung und Unterstützung durch eine_n Mentor_ in oder eine Peergroup umfasst, beinhaltet das Modul Training verschiedene Qualifikationsangebote zu laufbahnrelevanten Themen. Im Rahmen des Moduls Networking können neue Kontakte geknüpft sowie Netzwerkstrukturen innerhalb des Wissenschaftssystems ausgebaut werden. Nicht nur in der Kombination, sondern insbesondere in der Komplementarität der drei Module liegen große Potentiale: Neben dem Transfer von Erfahrungen und Wissen können vielschichtige Lernprozesse initiiert und damit unterschiedliche Schlüsselkompetenzen entwickelt werden.

Diese drei zentralen Bausteine von Mentoring-Programmen in der Wissenschaft werden im Folgenden detailliert beschrieben. 